\title{
A Relação entre o Processamento Fonológico e a Habilidade de Leitura: Evidência da Síndrome de Down e da Síndrome de Williams
}

\author{
The Relationship between Phonological Processing and Reading Ability: \\ Evidence from Down Syndrome and Williams Syndrome
}

\author{
Cláudia Cardoso-Martinss ${ }^{*} \&$ Juliane Ribeiro da Silva ${ }^{b}$ \\ ${ }^{a}$ Universidade Federal de Minas Gerais, Belo Horizonte, Brasil \\ ${ }^{b}$ Prefeitura de Belo Horizonte- Secretaria Municipal de Saúde, Belo Horizonte, Brasil
}

\begin{abstract}
Resumo
O presente artigo apresenta uma avaliação dos resultados de estudos que investigaram a relação entre o processamento fonológico e a habilidade de ler palavras em dois distúrbios do desenvolvimento: a síndrome de Down (SD) e a síndrome de Williams (SW). Os resultados sugerem que, como crianças sem transtornos do desenvolvimento, indivíduos com SD e indivíduos com SW aprendem a ler através do processamento e armazenamento de relações entre as letras e os sons nas palavras. É possível, no entanto, que processos não fonológicos desempenhem um papel mais importante na aprendizagem da leitura na SD e na SW do que entre crianças com desenvolvimento típico. Conforme é discutido no texto, há evidência de que variações em habilidades não verbais correlacionam-se mais for temente com a habilidade de leitura entre indivíduos com SD e indivíduos com SW do que entre crianças com desenvolvimento típico da leitura.

Palavras-chave: Leitura de palavras; processamento fonológico; síndrome de Down; síndrome de Williams.

Abstract

The present article presents a review of studies which investigated the relationship between phonological processing skills and the ability to read words in two developmental disorders: Down syndrome (DS) and Williams syndrome (WS). Results suggest that, like children without developmental disorders, individuals with DS and individuals with WS learn to read by processing and remembering letter-sound relations in words. It is nonetheless possible that non-phonological processes play a more important role in reading skills among individuals with DS and WS than among typically developed children. As discussed in the text, there is evidence that variations in non-verbal abilities are more strongly correlated with reading ability in DS and WS than among children with typical reading development

Keywords: Word reading; phonological processing; Down syndrome; Williams syndrome.
\end{abstract}

Os resultados de estudos realizados nas últimas três décadas sugerem que o processamento fonológico desempenha um papel crucial na aprendizagem da leitura em um sistema de escrita alfabético (ver, por exemplo, Snowling, 2004; ver Barrera \& Maluf, 2003; Capovilla, Gütschow \& Capovilla, 2004; Cardoso-Martins, 1991, 1995; Maluf \& Barrera, 1997; Salles \& Parente, 2002, para resultados de estudos investigando a relação entre o processamento fonológico e a habilidade de leitura de crianças aprendendo a ler em português). Uma explicação óbvia para esses resultados é que o processamento fonológico é necessário para a habilidade de ler através da recodificação das letras ou grupos de letras em seus sons correspondentes. Essa habilidade, por sua vez, é a chave

\footnotetext{
* Endereço para correspondência: Universidade Federal de Minas Gerais, Av. Antônio Carlos, 6627, Belo Horizonte, MG, 31270-901. Tel.: 3499 6270; Fax: 3499 5042. E-mail: cacau@fafich.ufmg.br
}

da habilidade de ler palavras fluentemente. De acordo com Ehri (1998), por exemplo, o processamento das relações letra-som nas palavras possibilita ao leitor armazenar seqüências de letras para milhares de palavras e, dessa maneira, recuperar a sua pronúncia e significado de forma acurada e automática ao ver a sua grafia impressa.

Pelo menos três habilidades fonológicas estão envolvidas na leitura de palavras através da recodificação fonológica (Wagner \& Torgesen, 1987): (a) a consciência fonológica, isto é, a consciência dos sons que constituem as palavras que ouvimos e falamos; (b) a codificação fonológica na memória de trabalho, necessária para o armazenamento temporário dos sons correspondentes às letras ou grupos de letras nas palavras; e, finalmente, (c) a recuperação dos códigos fonológicos correspondentes às letras ou grupos de letras na grafia da palavra. Há evidência de que variações em todas essas habilidades correlacionamse estreitamente com variações na habilidade de leitura de crianças com desenvolvimento típico, independentemente 
do efeito de diferenças na inteligência e no nível sócioeconômico da criança (ver, por ex., Snowling, 2004; Wagner \& Torgesen, 1987).

O presente artigo apresenta uma revisão de estudos que investigaram a relação entre o processamento fonológico e a habilidade de ler palavras em duas alterações do desenvolvimento comumente associadas à deficiência mental: a síndrome de Down (SD) e a síndrome de Williams (SW). Essas síndromes oferecem um contraste interessante para a investigação do papel desempenhado pelo processamento fonológico na habilidade de ler palavras. A razão para isso resulta do fato de que indivíduos com SD e SW apresentam padrões muito diferentes de habilidade verbal. Enquanto a linguagem constitui a área ou domínio mais comprometido na SD, ela é surpreendentemente bem desenvolvida na SW e certamente superior do que na $\mathrm{SD}$, sobretudo entre adolescentes e adultos (Karmiloff \& Karmiloff-Smith, 2002). Na medida em que a linguagem, em geral, e o processamento fonológico, em particular, desempenham um papel importante na aprendizagem da leitura, indivíduos com SW deveriam, portanto, apresentar um nível mais avançado de leitura de palavras do que indivíduos com SD (Snowling \& Hulme, 2005).

Conforme descrevemos a seguir, não encontramos nenhum estudo que tenha investigado essa hipótese diretamente. De fato, os estudos sobre a habilidade de leitura na SD e na SW são relativamente raros. No Brasil, por exemplo, não localizamos nenhum estudo que tenha investigado a habilidade de leitura na SW e, tanto quanto temos conhecimento, apenas três estudos (CardosoMartins \& Frith, 1999, 2001; Cardoso-Martins, Michalick \& Pollo, 2002) investigaram a relação entre o processamento fonológico e a habilidade de leitura na SD. Esperamos, portanto, que a presente revisão da literatura estimule a investigação da aquisição da leitura e da escrita na $\mathrm{SD}$ e na SW, assim como em outros distúrbios do desenvolvimento.

A presente revisão está dividida em três partes. Na primeira, apresentamos brevemente o modelo triangular de leitura de palavras e sua relação com as habilidades de processamento fonológico. $\mathrm{Na}$ segunda, apresentamos os resultados de estudos que investigaram a relação entre o processamento fonológico e a habilidade de leitura e escrita na SD e na SW. Na terceira parte, comparamos os resultados dos estudos com portadores da SD com os resultados dos estudos com portadores da SW à luz do modelo triangular de leitura.

\section{O Modelo Triangular de Leitura de Palavras}

Existem duas classes principais de modelos de leitura de palavras: os modelos de dupla-rota (por exemplo, Coltheart, 2005) e os modelos conexionistas (por exemplo, Plaut, 2005). A seguir, descrevemos brevemente o modelo conexionista de Seidenberg e McClelland (1989; ver também Harm \& Seidenberg, 1999), também conhecido pelo nome de modelo triangular da leitura (ver Figura 1). Conforme Bishop e Snowling (2004) observam, a pos- sibilidade de simular o processo de aprendizagem torna os modelos conexionistas particularmente adequados ao estudo do desenvolvimento da leitura.

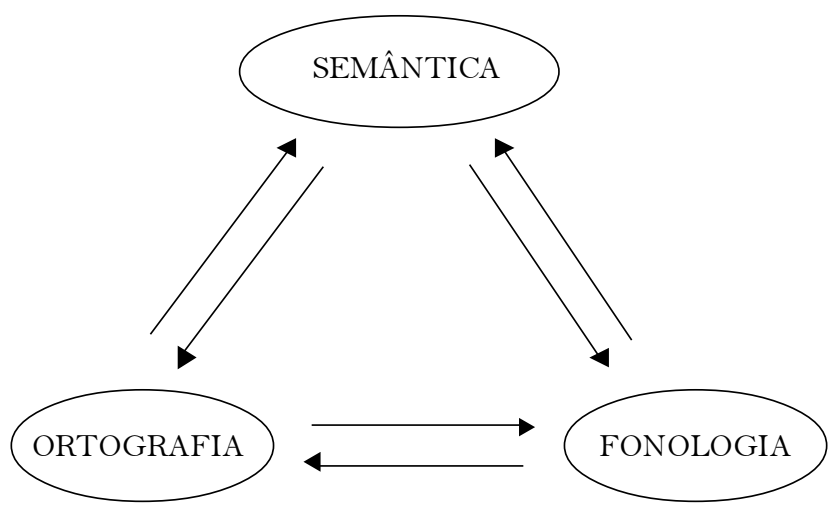

Figura 1. O Modelo triangular de leitura de palavras segundo Seidenberg e McClelland (1989).

De acordo com o modelo triangular, o reconhecimento de palavras escritas envolve a ativação de conexões entre três classes de representações: ortográficas, fonológicas e semânticas. Como nos modelos de dupla-rota, é possível discernir duas «vias» para a leitura de palavras em voz alta: a via fonológica, responsável pelo mapeamento entre as letras e os sons nas palavras, e a via semântica, em que o mapeamento entre a grafia e a fonologia da palavra é mediado semanticamente. Ao contrário dos modelos de dupla-rota, no entanto, no modelo triangular o reconhecimento de palavras resulta da ativação de todas as três classes de representações. Isso não significa, no entanto, que todas elas contribuam igualmente para o reconhecimento de palavras. Pelo contrário, o grau de ativação das representações fonológicas e semânticas parece variar em função de características da palavra e do leitor. Por exemplo, há evidência de que, nas fases iniciais da alfabetização, as representações fonológicas contribuem mais para o reconhecimento de palavras do que as representações semânticas (Snowling \& Hulme, 2005).

Tendo em vista que o desenvolvimento da memória fonológica e do vocabulário é superior na SW do que na SD (Mervis \& Morris, 2007), não seria surpreendente se indivíduos com SW também apresentassem um desempenho relativamente superior em tarefas que avaliam a habilidade de leitura de palavras. A seguir, apresentamos uma revisão de estudos que investigaram os correlatos da habilidade de leitura de palavras na SD e na SW com o intuito de avaliar essa hipótese.

\section{Processamento Fonológico e Leitura de Palavras na SD e na SW}

\section{Sindrome de Down}

A SD, ou Trissomia do 21, é uma anomalia cromossômica caracterizada pela presença de um cromossomo 21 extra. É a causa mais comum de deficiência mental, representando um terço da população com déficit intelectual. Confor- 
me observamos anteriormente, a linguagem é especialmente deficiente na SD. Com efeito, há evidência de que o desenvolvimento da linguagem é inferior até mesmo ao que seria esperado com base no nível de desenvolvimento mental da criança. Por outro lado, as habilidades viso-espaciais são condizentes com ou, até mesmo, superiores ao nível de desenvolvimento mental característico da síndrome (Pennington, Moon, Edgin, Stedron \& Nadel, 2003).

Segundo Buckley (1985), em torno de $40 \%$ dos jovens com síndrome de Down adquirem pelo menos alguma habilidade de leitura. Em alguns casos, a habilidade de leitura é excepcional. Por exemplo, Buckley cita casos de crianças com SD em idade pré-escolar que eram capazes de ler centenas de palavras. Tendo em vista as dificuldades de linguagem características da síndrome, Buckley sugeriu que as crianças com SD baseiam-se em suas capacidades viso-espaciais para aprender a ler. O fato de que algumas das crianças observadas por ela tenham começado a ler quando ainda mal podiam falar parece, de fato, consistente com a hipótese de que crianças com SD aprendem a ler visualmente. Conforme discutimos a seguir, no entanto, há evidência de que a habilidade de ler palavras na SD correlaciona-se estreitamente com medidas do processamento fonológico.

Cossu, Rossini e Marshall (1993) foram provavelmente os primeiros pesquisadores a investigar a relação entre o processamento fonológico e a habilidade de leitura na SD. Seu estudo incluiu 10 crianças italianas portadoras de SD cuja idade variava entre 8 e 15 anos $($ QI Médio $=44)$. Dez crianças típicas (Idade Média = 7;03 anos), emparelhadas às crianças com SD em função da habilidade de leitura, participaram como controles. Além de testes de inteligência e de leitura de palavras e pseudopalavras, ou seja, palavras que não existem e que, portanto, só podem ser lidas através da recodificação fonológica, todas as crianças foram submetidas a testes que avaliavam a habilidade de análise e síntese de segmentos fonológicos.

As crianças com SD apresentaram um desempenho muito pobre e significativamente inferior ao dos controles em todas as tarefas de consciência fonológica. Tendo em vista que as crianças com SD eram tão capazes de ler palavras e pseudopalavras quanto as crianças do grupo controle, Cossu et al. (1993) argumentaram que a consciência fonológica não é necessária para a aprendizagem da leitura em um sistema alfabético. É possível, no entanto, que as crianças com SD não tenham sido capazes de executar os testes de consciência fonológica, não porque não tivessem consciência dos segmentos fonológicos da fala, mas sim por não haverem compreendido as instruções (Bertelson, 1993; Byrne, 1993; Cardoso-Martins \& Frith, 2001). De fato, tarefas de análise e síntese de fonemas são difíceis para crianças em idade pré-escolar, independentemente de sua habilidade de leitura (Scholes, 1991). Uma vez que as crianças com SD que participaram do estudo de Cossu et al. (1993) tinham, em média, apenas 5 anos de idade mental, não é surpreendente que houvessem apresentado dificuldade nas tarefas utilizadas para avaliar a consciência fonológica.
Os resultados do estudo de Fowler, Dohert e Boynton (1995) são condizentes com essa interpretação. Essas pesquisadoras avaliaram a relação entre a habilidade de leitura e a habilidade de segmentar fonemas em uma amostra de 33 indivíduos com SD, entre 17 e 25 anos de idade, os quais já haviam começado a ler. Relativamente às crianças que participaram do estudo de Cossu et al. (1993), os participantes do estudo de Fowler et al. apresentavam um nível de desenvolvimento intelectual elevado. Por exemplo, sua idade mental, avaliada através de um teste de vocabulário receptivo - o PPVT-R (Dunn \& Dunn, 1981) era, em média, de 8 anos. Talvez em função disso, tenham apresentado um desempenho relativamente superior no teste utilizado para avaliar a habilidade de segmentação fonêmica. Além disso, o desempenho naquele teste correlacionou-se significativamente com a habilidade de ler palavras e pseudopalavras. De fato, mesmo após o controle do efeito de diferenças no teste de vocabulário e em um teste de inteligência, variações na habilidade de segmentar fonemas contribuíram significativamente para a habilidade de leitura de palavras e pseudopalavras, sugerindo que a consciência fonológica é um correlato importante da habilidade de leitura na SD. Resultados semelhantes foram obtidos para uma tarefa de memória verbal e, embora de forma menos acentuada, para uma tarefa de recuperação lexical.

Assim como Fowler et al. (1995), vários estudiosos têm encontrado correlações estreitas entre medidas do processamento fonológico e medidas da habilidade de leitura de palavras na SD (Cardoso-Martins \& Frith, 2001; Cardoso-Martins, Robinson, Olson \& Pennington, in press; Cupples \& Iacono, 2000; Fletcher \& Buckley, 2002; Gombert, 2002; Kennedy \& Flynn, 2003; Laws, 1998; Laws \& Gunn, 2002; Snowling, Hulme \& Mercer, 2002). Apenas os resultados dos estudos conduzidos por CardosoMartins e Frith, Gombert (2002) e Snowling et al. (2002) são descritos a seguir. Ao contrário dos demais, esses estudos incluíram um grupo de crianças típicas, emparelhadas aos indivíduos com SD em função da habilidade de leitura de palavras. Esse controle é importante, pois reduz a possibilidade de que diferenças entre os dois grupos possam ser atribuídas a diferenças na sua habilidade de leitura.

O estudo de Cardoso-Martins e Frith (2001) envolveu 33 crianças e adultos brasileiros com SD, com idade entre 10 e $49 \operatorname{anos}(M=23$ anos). Todos, sem exceção, já haviam começado a ler por ocasião do estudo. Um grupo de 33 crianças com desenvolvimento típico, emparelhadas aos indivíduos com SD em função da habilidade de leitura de palavras e pseudopalavras, também participou do estudo. Sua idade variava entre 6 e 9 anos $(M=7$ anos). Os participantes foram submetidos a testes de inteligência, conhecimento do nome das letras e, finalmente, a duas tarefas de consciência fonêmica. A tarefa de subtração de fonema era semelhante a uma das tarefas utilizadas por Cossu et al. (1993). Nessa tarefa, o participante devia repetir uma palavra enunciada pelo examinador sem o fonema inicial. $\mathrm{Na}$ tarefa de detecção de fonema, por outro lado, o participante devia simplesmente identificar, entre três palavras 
diferentes, aquela que começava com um fonema enunciado pelo pesquisador.

Embora os participantes com SD tenham apresentado um desempenho inferior ao dos controles em ambas as tarefas de consciência fonêmica, seu desempenho na tarefa de detecção de fonema foi muito bom. De fato, 30 dos 33 indivíduos com SD apresentaram escores acima do que seria esperado caso estivessem respondendo ao acaso, não diferindo, nessa análise, dos controles. Além disso, em ambos os grupos, os escores nas tarefas de consciência fonológica correlacionaram-se significativamente com a habilidade de leitura de palavras e pseudopalavras. De fato, os índices de correlação entre a tarefa de subtração de fonemas e as tarefas de leitura foram muito semelhantes nos dois grupos. Finalmente, conforme ocorreu para as crianças do grupo controle, o desempenho na tarefa de subtração de fonemas correlacionou-se com a habilidade de leitura, mesmo após o controle do efeito de variações na inteligência não-verbal e no conhecimento do nome das letras. No entanto, após esse controle, a contribuição da consciência de fonemas foi maior para os controles do que para os indivíduos com SD. Por outro lado, variações na medida de inteligência não-verbal contribuíram mais para as diferenças na habilidade de leitura entre os indivíduos com SD do que entre as crianças do grupo controle.

Gombert (2002) avaliou a consciência fonológica de 11 crianças francesas com SD (Idade média = 13;09 anos; QI médio $=47)$. Onze crianças típicas (Idade média $=7$ anos), com habilidade de leitura semelhante à das crianças com $\mathrm{SD}$, também participaram do estudo. Várias tarefas foram utilizadas para avaliar a consciência fonológica. O grupo com SD apresentou um desempenho significativamente inferior ao desempenho do grupo controle em todas elas. Como no estudo de Cardoso-Martins e Frith (2001), no entanto, a habilidade de manipular e segmentar fonemas correlacionou-se significativamente com a habilidade de ler palavras e pseudopalavras em ambos os grupos de participantes.

Snowling et al. (2002) realizaram uma série de estudos com o objetivo de avaliar a relação entre diferentes medidas de consciência fonológica e a habilidade de leitura na SD. O primeiro deles envolveu 29 crianças com SD entre 6 e 17 anos, cuja idade de leitura variava entre 5 e 12 anos. Trinta e uma crianças típicas (Idade média $=5 ; 03$ anos) também participaram do estudo. Sua habilidade de leitura de palavras era semelhante à habilidade das crianças com $\mathrm{SD}$, variando entre 5;00 e 9;04 anos. A consciência fonológica foi avaliada através de tarefas que pressupunham a habilidade de prestar atenção consciente a diferentes segmentos fonológicos.

Como nos estudos relatados anteriormente, a sensibilidade ao segmento fonêmico da fala correlacionou-se significativamente com a habilidade de ler palavras e pseudopalavras, em ambos os grupos de crianças. Com efeito, as correlações entre a medida de consciência fonêmica e as medidas de habilidade de leitura foram praticamente idênticas para os dois grupos. No entanto, ao contrário dos resultados obtidos por Cardoso-Martins e Frith (2001), a consciência de fonemas não mais contribuiu significativamente para a variação na habilidade de ler palavras e pseudopalavras, após o controle do efeito de variações na idade e no vocabulário. Além disso, entre as crianças com $\mathrm{SD}$, o conhecimento dos sons das letras não se correlacionou com a habilidade de leitura de palavras ou pseudopalavras e nem tampouco com as medidas de consciência fonológica.

Snowling et al. (2002) basearam-se nesses resultados para sugerir que indivíduos com SD baseiam-se menos nas habilidades fonológicas para aprender a ler do que crianças com desenvolvimento típico. É verdade que o conhecimento do nome e dos sons das letras correlacionouse especificamente com a habilidade de leitura e a consciência fonológica apenas entre as crianças típicas. No entanto, conforme ocorreu com as crianças com SD, a consciência de fonemas não contribuiu para as variações na habilidade de leitura das crianças típicas, após o controle do efeito de variações na idade e no vocabulário. Além disso, em ambos os grupos, a habilidade de ler palavras correlacionou-se significativamente com a habilidade de ler pseudopalavras, independentemente de variações no vocabulário e na idade das crianças. Esses resultados sugerem, portanto, que indivíduos com SD baseiam-se em processos fonológicos para aprender a ler. É possível, no entanto, que tenham dificuldade em fazer uso daqueles processos para aprender a ler. Isso explicaria os resultados negativos encontrados por Snowling et al. para o conhecimento dos sons das letras entre as crianças com SD que participaram do seu estudo.

Além da tarefa de consciência fonêmica, os participantes do estudo de Snowling et al. (2002) foram submetidos a tarefas que avaliam a sensibilidade à rima e à sílaba. As crianças com SD apresentaram um desempenho significativamente inferior ao dos controles em praticamente todas as tarefas de consciência fonológica. No entanto, após o controle do efeito de diferenças no vocabulário receptivo, apenas a diferença em uma tarefa que avaliava a sensibilidade à rima manteve-se significativa. Esses resultados são surpreendentes, tendo em vista que, entre crianças com desenvolvimento típico, tarefas que avaliam a sensibilidade à rima são em geral mais fáceis do que tarefas que avaliam a sensibilidade ao fonema (Stanovich, Cunningham \& Cramer, 1984; Yopp, 1988).

Snowling et al. (2002) conduziram dois estudos adicionais para explorar esses resultados. Um deles envolveu 23 crianças com SD (Idade média = 13 anos) e 34 crianças típicas (idade media $=5 ; 04$ anos). Embora o grupo de crianças com SD tenha apresentado um desempenho significativamente inferior ao das crianças típicas em um teste de detecção de rima, os dois grupos não diferiram significativamente em relação à habilidade de detectar fonema.

Ao contrário dos estudos anteriores, o mesmo procedimento metodológico foi utilizado para avaliar a habilidade de detectar rima e fonema no terceiro e último estudo conduzido por Snowling et al. (2002). A criança escutava um par de palavras e devia dizer se as palavras rimavam ou não, no caso do teste de rima, e se começavam ou não com o mesmo som, no caso do teste de fonema. Trinta 
crianças típicas (Idade média $=5 ; 08$ anos) e 30 crianças com SD (Idade média = 13;04 anos) serviram como participantes. Mais uma vez, os resultados sugerem que a habilidade de detectar rima é difícil na SD. Embora ambos os grupos tenham achado a tarefa de detecção de fonema mais fácil do que a tarefa de detecção de rima, as crianças com SD, mas não as crianças do grupo controle, responderam ao acaso no teste de rima. Além disso, como os controles, as crianças com SD apresentaram um desempenho superior ao que seria esperado caso estivessem respondendo ao acaso no teste de detecção de fonemas.

Outros estudiosos (Cardoso-Martins \& Frith, 1999; Cardoso-Martins et al., 2002; Gombert, 2002) também observaram uma maior dificuldade em tarefas de detecção de rima do que em tarefas de detecção de fonema na SD. Cardoso-Martins et al. mostraram que essa dificuldade caracteriza tanto leitores quanto não-leitores com SD. O estudo dessas pesquisadoras envolveu dois grupos de brasileiros com SD: 39 leitores entre 8 e 35 anos, e 30 não-leitores entre 6 e 20 anos. Cento e trinta crianças típicas também participaram do estudo: 52 leitores, entre 5 e 10 anos de idade, e 78 crianças que ainda não haviam começado a ler e cuja idade variava entre 3 e 5 anos. Os participantes foram submetidos a uma tarefa de detecção de rima e a duas tarefas de detecção de fonema.

Os indivíduos com SD acharam a tarefa de detecção de rima significativamente mais difícil do que ambas as tarefas de detecção de fonema. Isso ocorreu tanto entre os leitores quanto entre os não-leitores. Por outro lado, os controles que ainda não haviam começado a ler acharam a tarefa de detecção de rima mais fácil do que as tarefas de detecção de fonema, enquanto aqueles que já haviam começado a ler acharam ambos os tipos de tarefa igualmente fáceis. Esses resultados confirmam os resultados de Snowling et al. (2002) e sugerem que, ao contrário do que parece ocorrer entre crianças típicas, a sensibilidade à rima não parece ser um precursor da sensibilidade ao fonema na SD.

Em suma, há evidência de que indivíduos com SD apresentam dificuldades em tarefas que avaliam o processamento fonológico. Isso não significa, no entanto, que a habilidade de leitura independa do processamento fonológico na SD. Pelo contrário, os resultados dos estudos descritos anteriormente sugerem que, semelhantemente ao que ocorre entre crianças sem alterações do desenvolvimento, as habilidades de processamento fonológico desempenham um papel importante na aprendizagem da leitura de palavras na SD. É possível, entretanto, que, em virtude de seus déficits fonológicos, indivíduos com SD tenham mais dificuldade em aprender a ler através da recodificação fonológica do que o que ocorre entre crianças com desenvolvimento típico (Snowling et al., 2002). Em outras palavras, é possível que, relativamente a crianças típicas do mesmo nível de leitura, indivíduos com SD baseiem-se mais em processos não fonológicos para aprender a ler. Isso explicaria os escores relativamente baixos desses indivíduos em tarefas de consciência fonológica, assim como os resultados do estudo de Cardoso-Martins e Frith (2001). Conforme descrevemos anteriormente, Cardoso-Martins e Frith encontraram uma correlação mais acentuada entre a inteligência não-verbal e a habilidade de ler palavras e pseudopalavras entre os participantes com SD do que entre os leitores típicos. Uma tarefa importante para a pesquisa futura consistirá em investigar a natureza das habilidades não-verbais envolvidas na aprendizagem da leitura na SD.

\section{Síndrome de Williams}

A SW é uma alteração neurogenética rara que resulta de uma pequena deleção no braço longo do cromossomo 7 (Hillier et al., 2003). Ao contrário do que ocorre na SD, a habilidade verbal é surpreendentemente bem desenvolvida na SW em comparação com a cognição viso-espacial. É possível, portanto, que indivíduos com SW aprendam a ler palavras relativamente bem (Snowling \& Hulme, 2005). Com efeito, Pagon et al. (1987; citado em Howlin, Davies \& Udwin, 1998) sugeriram que a leitura é a área mais forte do desempenho escolar de indivíduos com SW. De fato, todos os nove leitores com SW que participaram do seu estudo apresentaram habilidade de leitura superior ao que seria esperado com base em seu nível de desenvolvimento intelectual.

Outros pesquisadores, no entanto, têm relatado resultados menos otimistas. Por exemplo, Howlin et al. (1998) avaliaram a habilidade de leitura e escrita de 67 indivíduos com SW, cuja idade variava entre 19 e 39 anos. Entre os 47 participantes que já haviam começado a ler, a habilidade de leitura e escrita de palavras foi inferior ao nível que seria esperado com base na inteligência verbal, avaliada através de um teste de vocabulário receptivo. Resultados semelhantes foram relatados por Levy, Smith e Tager-Flusberg (2003) em relação à habilidade de ler pseudopalavras. Embora os 17 indivíduos com SW que participaram do seu estudo tivessem apresentado, em média, idade mental equivalente a 9;04 anos, seu desempenho no teste de leitura de pseudopalavras foi semelhante ao de uma criança típica de 8;02 anos.

Relativamente poucos estudos investigaram a relação entre a leitura e o processamento fonológico na SW. Desses, apenas os estudos realizados por Laing, Hulme, Grant e Karmiloff-Smith (2001) e Menghini, Verucci e Vicari (2004) incluíram um grupo de controle.

Quinze indivíduos ingleses portadores de SW (Idade Média $=15 ; 01$ anos) e 15 crianças típicas (Idade Média $=$ 6;09 anos), individualmente emparelhados em função do seu desempenho em um teste de vocabulário receptivo e um teste de leitura de palavras, participaram do estudo de Laing et al. (2001). O processamento fonológico foi avaliado através de tarefas de consciência fonológica, tarefas de memória verbal de curto prazo e, finalmente, tarefas de nomeação rápida de figuras e dígitos. Embora o grupo controle tenha apresentado um desempenho superior em todas as tarefas, as diferenças não foram, em geral, estatisticamente significativas. Além disso, as medidas de consciência fonológica correlacionaram-se com a habilidade de leitura em ambos os grupos. Entretanto, após o 
controle do efeito de diferenças individuais na inteligência verbal e não-verbal, e na idade cronológica, variações na consciência fonológica não mais contribuíram significativamente para as variações na habilidade de leitura dos indivíduos com SW.

Menghini et al. (2004), por outro lado, encontraram resultados positivos para a habilidade de manipular sílabas em um estudo com 16 indivíduos italianos com SW, cuja idade variava entre 10 e 30 anos $(M=17 ; 07$ anos $)$. Além dos indivíduos com SW, o estudo contou com a participação de 16 crianças típicas entre 6 e 8 anos, emparelhadas aos indivíduos com SW em função da idade mental (Idade mental média $=7 ; 00$ e 7;07 anos para os indivíduos com e sem SW, respectivamente). A consciência fonológica foi avaliada através de testes de segmentação e manipulação de sílabas e de um teste de detecção de rima.

De acordo com Menghini et al. (2004), a tarefa de deleção de sílabas correlacionou-se significativamente com a habilidade de leitura de palavras e pseudopalavras na SW, independentemente de variações na idade mental. Entre as crianças do grupo controle, por outro lado, após o controle do efeito de diferenças na idade mental, variações na segmentação de sílabas correlacionaram-se apenas com a habilidade de leitura de pseudopalavras.

Em resumo, o processamento fonológico parece desempenhar um papel importante na habilidade de leitura na SW. Por exemplo, no estudo de Laing et al. (2001), a habilidade de ler palavras correlacionou-se significativamente com a habilidade de ler pseudopalavras. Com efeito, a habilidade de ler através da recodificação fonológica continuou a contribuir significativamente para a habilidade de leitura de palavras na SW, mesmo após o controle de variações na idade e no vocabulário receptivo. O mesmo ocorreu para as medidas de nomeação rápida de objetos e dígitos. Além disso, no estudo de Menghini et al. (2004), a habilidade de manipular sílabas correlacionou-se com a habilidade de leitura, independentemente de diferenças na idade mental.

É possível que diferenças nos sistemas de escrita tenham, pelo menos em parte, contribuído para as diferenças encontradas por Laing et al. (2001) e Menghini et al. (2004) no que diz respeito à importância relativa da consciência fonológica e da inteligência na SW. Segundo Jiménez, Siegel e López (2003), quanto mais irregular é o sistema de escrita, maior a importância da inteligência. Isso explicaria a contribuição relativamente grande da inteligência no estudo de Laing et al. Os participantes do estudo de Laing et al. estavam aprendendo a ler em inglês, uma ortografia sabidamente mais irregular do que a ortografia italiana.

\section{A Habilidade de Leitura na SD e na SW}

Os resultados dos estudos revisados apóiam a expectativa de que, semelhantemente ao que parece ocorrer entre crianças com desenvolvimento típico, o processamento fonológico desempenha um papel importante na aprendizagem da leitura na SW e na SD. Conforme discutido a seguir, por outro lado, não encontramos evidência de que a habilidade de ler palavras é mais desenvolvida na SW do que na SD.

Não localizamos nenhum estudo que tenha investigado essa questão diretamente. Em virtude disso, comparamos os resultados obtidos para portadores da SD e da SW em diferentes estudos. Dois pares de estudos foram incluídos nessa comparação: um envolvendo indivíduos falantes do italiano e o outro, indivíduos falantes do inglês. A comparação da habilidade de leitura em inglês envolveu os jovens e adultos com $\mathrm{SD}(N=33$; idade cronológica entre 17 e 25 anos) que participaram do estudo de Fowler et al. (1995) e os jovens e adultos com SW que participaram do estudo de Howlin et al. (1998) e que já haviam começado a ler ( $N=47$; idade cronológica entre 19 e 39 anos). Por sua vez, a comparação da habilidade de leitura em italiano envolveu as crianças com $\mathrm{SD}(N=10$, idade cronológica entre 8 e 15 anos) e os jovens e adultos com SW $(N=16$, idade cronológica entre 10 e 30 anos) que participaram do estudo de Cossu et al. (1993) e do estudo de Menghini et al. (2004), respectivamente.

Os resultados dessas comparações sugerem que a habilidade de leitura de palavras é muito semelhante na SW e na SD. Por exemplo, a idade equivalente de leitura foi, em média, igual a 8;05 anos para os indivíduos com SD que participaram do estudo de Fowler et al. (1995) e 8;08 anos para os indivíduos com SW que participaram do estudo de Howlin et al. (1998).

Resultados análogos foram encontrados para as comparações envolvendo as crianças e os jovens com SD e SW aprendendo a ler em italiano. No estudo de Cossu et al. (1993), as crianças com SD leram, em média, 94\% das palavras reais e $88 \%$ das pseudopalavras. Os números correspondentes para os jovens com SW que participaram do estudo de Menghini et al. (2004) foram 90\% e $76 \%$.

Naturalmente, essas comparações devem ser interpretadas com cuidado, tendo em vista que testes diferentes foram utilizados para avaliar as habilidades fonológicas e a habilidade de leitura nos diversos estudos. Além disso, não é certo que os indivíduos envolvidos nesses estudos pertencessem a famílias de nível sócio-econômico equivalente ou que houvessem tido experiências educacionais semelhantes. Mesmo assim, os resultados não deixam de ser surpreendentes, sobretudo se levarmos em consideração que, em ambas as comparações, os indivíduos com SW eram mais velhos do que os indivíduos com SD e apresentavam um nível de inteligência relativamente superior.

Com base no modelo triangular de Seidenberg e McClelland (1989), déficits em qualquer uma das três classes de representações poderiam interferir de maneira negativa com o desenvolvimento da leitura de palavras. A seguir, examinamos a possibilidade de que o desenvolvimento inesperadamente baixo da leitura na SW possa ser atribuído a déficits nas representações semânticas, fonológicas e/ou ortográficas.

Alguns autores têm argumentado que, relativamente a crianças típicas do mesmo nível de desenvolvimento mental, portadores da SW apresentam déficits semânticos (ver, 
por exemplo, Temple, 2006). Com base nesse tipo de evidência, Snowling e Hulme (2005) sugeriram que as dificuldades semânticas características da SW poderiam dificultar o desenvolvimento da habilidade de ler palavras. Conforme discutimos a seguir, no entanto, é pouco provável que dificuldades semânticas na SW nos ajudem a compreender a aparente ausência de diferenças entre a habilidade de leitura na SD e na SW.

Dificuldades semânticas também estão presentes na SD e, ao que tudo indica, de forma mais acentuada do que na SW (Mervis \& Morris, 2007). De fato, os indivíduos com SD que participaram do estudo de Fowler et al. (1995) apresentaram escores inferiores aos dos indivíduos com SW que participaram do estudo de Howlin et al. (1998) em um teste muito semelhante de vocabulário receptivo. Nem por isso, contudo, a sua habilidade de leitura de palavras foi inferior. Além disso, alguns pesquisadores (por exemplo, Snowling \& Hulme, 2005) têm sugerido que as representações semânticas são mais importantes para a aprendizagem da grafia de palavras irregulares. Na medida em que isso é verdade, indivíduos com SW deveriam apresentar uma dificuldade especialmente mais acentuada na leitura de palavras irregulares do que na leitura de palavras regulares. Temple (2006) argumenta ter encontrado um padrão semelhante a esse em um estudo envolvendo oito indivíduos com SW e oito crianças típicas com o mesmo nível de habilidade de leitura. Por outro lado, no estudo de Menghini et al. (2004), a diferença favorecendo a leitura de palavras regulares foi menos acentuada para os jovens com SW do que para os controles.

Déficits nas representações fonológicas tampouco parecem capazes de explicar a aparente ausência de diferenças entre a SW e a SD no que diz respeito à habilidade de ler palavras. É verdade que os resultados dos estudos descritos anteriormente sugerem a presença de dificuldades fonológicas na SW, as quais não podem ser explicadas em termos apenas de um déficit intelectual. Por exemplo, os indivíduos com SW que participaram do estudo de Laing et al. (2001) apresentaram um desempenho inferior ao dos controles no teste de abrangência de dígitos, apesar dos dois grupos apresentarem níveis semelhantes de desenvolvimento mental e de habilidade de leitura. Além disso, no estudo de Howlin et al. (1998), os indivíduos com SW apresentaram, em média, um escore ponderado de apenas 2,7 no teste de abrangência de dígitos. De fato, esse foi o menor escore ponderado na escala WAIS-R. Finalmente, embora os indivíduos com SW que participaram do estudo de Howlin et al. houvessem apresentado um desempenho semelhante ao dos controles na leitura de palavras reais, sua habilidade de ler pseudopalavras foi significativamente inferior. Se, por um lado, esses resultados sugerem que indivíduos com SW apresentam dificuldades em tarefas de processamento fonológico, toda a evidência disponível sugere que o processamento fonológico é superior na SW do que na SD (Mervis \& Morris, 2007).

Em termos do modelo triangular, portanto, resta-nos apenas a possibilidade de déficits nas representações or- tográficas como explicação da habilidade inesperadamente baixa de leitura de palavras na SW. É possível que, ao contrário do que parece ocorrer em relação às representações fonológicas e semânticas, indivíduos com SW tenham dificuldades ortográficas mais acentuadas do que indivíduos com SD. Por exemplo, é possível que suas dificuldades viso-espaciais interfiram com a habilidade de distinguir letras como $b$ e $d, b$ e $p, d$ e $q$, etc., que diferem apenas em relação à orientação espacial. Essa dificuldade, por sua vez, poderia dar origem a representações ortográficas mais imprecisas e instáveis.

Os índices relativamente altos de correlação entre a leitura e a inteligência na SD e na SW sugerem fortemente que o desenvolvimento da leitura de palavras é inesperadamente baixo em ambas as síndromes (ver CardosoMartins et al., in press, para uma discussão dessa questão na SD). Conforme discutimos acima, as dificuldades de leitura na SD e na SW podem ser explicadas em termos de déficits nas representações fonológicas, semânticas e ortográficas. Embora seja provável que indivíduos com SD e SW apresentem déficits em todas as três classes de representação, tudo indica que a gravidade dos déficits fonológicos seja maior na SD do que na SW. Por outro lado, é possível que indivíduos com SW tenham mais dificuldades com as representações or tográficas do que indivíduos com SD. Estudos futuros, envolvendo indivíduos com SD e SW emparelhados em função do nível de inteligência e do nível de leitura, deverão investigar essa possibilidade.

Além de importantes do ponto de vista prático, os resultados desses estudos serão importantes do ponto de vista teórico. A pesquisa nos últimos anos tem destacado a importância da fonologia para a aprendizagem da leitura. Com efeito, Perfetti e Dunlap (in press) sugeriram que uma premissa central de uma teoria universal da leitura deverá ser o princípio fonológico, segundo o qual a leitura de palavras ativa a fonologia em todas as línguas. Os resultados discutidos neste artigo sugerem que as representações fonológicas também são ativadas em todos os leitores, independentemente de suas habilidades fonológicas. Contudo, eles também sugerem que o funcionamento do princípio fonológico varia em função de variações em outras habilidades cognitivas relevantes. Estudos sobre a habilidade de leitura em diferentes transtornos do desenvolvimento contribuirão, certamente, para elucidar a natureza dessas habilidades e de como elas interagem com o funcionamento do princípio fonológico na aprendizagem da leitura.

\section{Referências}

Barrera, S. D., \& Maluf, M. R. (2003). Consciência metalinguística e alfabetização: Um estudo com crianças da primeira série do ensino fundamental. Psicologia Reflexão $e$ Crítica, 16(3), 491-502.

Bertelson, P. (1993). Reading acquisition and phonemic awareness testing: How conclusive are data from Down's syndrome? Cognition, 48, 281-283. 
Bishop, D. V. M., \& Snowling, M. J. (2004). Developmental dyslexia and specific language impairment: Same or different? Psychological Bulletin, 130, 858-886.

Buckley, S. (1985) Attaining basic educational skills: Reading, writing and number. In D. Lane \& B. Stratford (Eds.), Current approaches to Down's syndrome (pp. 315-343). New York: Praeger Press.

Byrne, B. (1993). Learning to read in the absence of phonemic awareness? A comment on Cossu, Rossini an Marshall (1993). Cognition, 48, 285-288.

Capovilla, A. G. S., Guitschow, C. R. D., \& Capovilla, F. C. (2004). Habilidades cognitivas que predizem competência de leitura e escrita. Psicologia Teoria e Prática, 6(2), 13-26.

Cardoso-Martins, C. (1991). Awareness of phonemes and alphabetic literacy acquisition. British Journal of Educational Psychology, 61, 164-173.

Cardoso-Martins, C. (1995). Sensitivity to rhymes, syllables and phonemes in literacy acquisition in Portuguese. Reading Research Quarterly, 30, 808-828.

Cardoso-Martins, C., \& Frith, U. (1999). Consciência fonológica e habilidade de leitura na Síndrome de Down. Psicologia: Reflexão e Crítica, 12(1), 209-224

Cardoso-Martins, C., \& Frith, U. (2001). Can individuals with Down syndrome acquire alphabetic literacy skills in the absence of phoneme awareness? Reading and Writing, 14, 361375.

Cardoso-Martins, C., Michalick, M. F., \& Pollo, T. C. (2002). Is sensitivity to rhyme a developmental precursor to sensitivity to phoneme?: Evidence from individuals with Down syndrome. Reading and Writing, 15, 439-454.

Cardoso-Martins, C., Robinson, R., Olson, D., \& Pennington, B. (in press). Component reading skills in Down syndrome. Reading and writing.

Coltheart, M. (2005). Modeling reading: The dual-route approach. In M. J. Snowling \& C. Hulme (Eds.), The science of reading: $A$ handbook (pp.6-23). Oxford, UK: Blackwell.

Cossu, G., Rossini, F., \& Marshall, J. C. (1993). When reading is acquired but phonemic awareness is not: A study of literacy in Down 's syndrome. Cognition, 46, 129-138.

Cupples, L., \& Iacono, T. (2000). Phonological awareness and oral reading skill in children with Down syndrome. Journal of Speech, Language, and Hearing Research, 43, 595-608.

Dunn, L., \& Dunn, L. (1981). Peabody Picture Vocabulary TestRevised. Circle Pines, MN: American Guidance Service.

Ehri, L. C. (1998). Learning to read and learning to spell are one and the same, almost. In C. Perfetti, L. Rieben \& M. Fayol (Eds.), Learning to spell: Research, theory and practice across languages (pp. 237-269). Mahwah, NJ: Erlbaum.

Fletcher, H., \& Buckley, S. (2002). Phonological awareness in children with Down syndrome. Down Syndrome Research and Practice, 8(1), 11-18.

Fowler, A. E., Dohert, B. J., \& Boynton, L. (1995). The basis of reading skill in young adults with Down syndrome. In $\mathrm{H}$. Nadel \& D. Rosenthal (Eds), Down Syndrome: Living and learning in the community (pp. 182-196). New York: Wiley-Liss.

Gombert, J. E. (2002). Children with Down syndrome use phonological knowledge in reading. Reading and writing, 15, $455-469$

Harm, M. W., \& Seidenberg, M. S. (1999). Phonology, reading acquisition, and dyslexia: Insights from connectionist models. Psychological Review, 106, 491-528.

Hillier, L. W., Fulton, R. S., Fulton, L. A., Graves, T. A., Pepin, K. H., Wagner-McPherson, C. et al. (2003). The DNA sequence of chromosome 7. Nature, 424, 157-164.
Howlin, P., Davies, M., \& Udwin, O. (1998). Cognitive functioning in adults with Williams syndrome. Journal of Child Psychology and Psychiatry, 39(2), 183-189.

Jiménez, J. E., Siegel, L. S., \& López, M. R. (2003). The relationship between IQ and reading disabilities in Englishspeaking Canadian and Spanish children. Journal of Learning. Disabilities, 36(1), 15-23.

Karmiloff, K., \& Karmiloff-Smith, A. (2002). Pathways to language: From fetus to adolescent. Cambridge, MA: Harvard University Press.

Kennedy, E. J., \& Flynn, M. C. (2003). Early phonological awareness and reading skills in children with Down syndrome. Down Syndrome Research and Practice, 8(3), 100-109.

Laing, E., Hulme, C., Grant, J., \& Karmiloff-Smith, A. (2001). Learning to read in Williams syndrome: Looking beneath the surface of atypical reading development. Journal of Child Psychology and Psychiatry, 42(6), 729-739.

Laws, G. (1998) The use of nonword repetition as a test of phonological memory in children with Down syndrome. Journal of Child Psychology and Psychiatry, 39(8), 1119-1130.

Laws, G., \& Gunn, D. (2002). Relationships between reading, phonological skills and language development in individuals with Down syndrome: A five year follow-up study. Reading and Writing, 15, 527-548.

Levy, Y., Smith, J., \& Tager-Flusberg, H. (2003). Word reading and reading-related skills in adolescents with Williams syndrome. Journal of Child Psychology and Psychiatry, 44(4), 576-587

Maluf, M. R., \& Barrera, S. D. (1997). Consciência fonológica e linguagem escrita em pré-escolares. Psicologia Reflexão e Crítica, $1 O(1), 125-145$.

Menghini, D., Verucci, L., \& Vicari, S. (2004). Reading and phonological awareness in Williams syndrome. Neuropsychology, 18(1), 29-37.

Mervis, C. B., \& Morris, C. A. (2007) Williams syndrome. In M. M. M. Mazzocco \& J. Ross (Eds.), Neurogenetic developmental disorders: Variation of manifestation in childhood (pp. 199-262). Cambridge, MA: MIT Press.

Pennington, B. F., Moon, J., Edgin, J., Stedron, J., \& Nadel, L. (2003). The neuropsychology of Down syndrome: Evidence for hippocampal dysfunction. Child Development, 74(1), 75-93.

Perfetti, C. A., \& Dunlap, S. (in press). Learning to read: General principles and writing system variations. In K. Koda \& A. Zehler (Eds.), Learning to read across languages. Mahwah, NJ: Erlbaum.

Plaut, D. C. (2005). Connectionist approaches to reading. In M. J. Snowling \& C. Hulme (Eds.), The science of reading: $A$ handbook (pp. 24-38). Oxford, UK: Blackwell.

Salles, J. F., \& Parente, M. A. M. P. (2002). Relação entre os processos cognitivos envolvidos na leitura de palavras e as habilidades de consciência fonológica em escolares. Pró-Fono Revista de Atualização Científica, 14(2), 175-186.

Scholes, R. (1991). Phoneme deletion and literacy in native and non-native speakers of English. Journal of Research in Reading, 14, $130-140$

Seidenberg, M. S., \& McClelland, J. L. (1989). A distributed, developmental model of word recognition. Psychological Review, 96, 523-568.

Snowling, M. J. (2004). Dislexia (2. ed.). São Paulo, SP: Santos. Snowling, M. J., \& Hulme, C. (2005). Learning to read with a language impairment. In M. J. Snowling \& C. Hulme (Eds.), The science of reading: $A$ handbook (pp. 397-412). Oxford, UK: Blackwell. 
Cardoso-Martins, C. \& Silva, J. R. da (2008). A Relação entre o Processamento Fonológico e a Habilidade de Leitura: Evidência da Síndrome de Down e da Síndrome de Williams.

Snowling, M. J., Hulme, C., \& Mercer, R. C. (2002). A deficit in rime awareness in children with Down syndrome. Reading and Writing, 15, 471-495.

Stanovich, K., Cunningham, A., \& Cramer, B. (1984). Assessing phonological awareness in kindergarten children: Issues of task comparability. Journal of Experimental Child Psychology, 38, 175-190

Temple, C. M. (2006). Developmental and acquired dyslexias. Cortex, 42(6), 898-910.

Wagner, R. K., \& Torgesen, J. K. (1987). The nature of phonological processing and its causal role in the acquisition of reading skills. Psychological Bulletin, 101(2), 192-212.

Yopp, H. (1988). The validity and reliability of phonemic awareness test. Reading Research Quarterly, 33, 159-177. 